\title{
AN EFFECTIVE MECHANISM FOR SECURING AND MANAGING PASSWORD USING AES-256 ENCRYPTION \& PBKDF2
}

\author{
Dr. Rajeshree Khande \\ Associate Head, School of Computer Science, MIT-World Peace University, \\ Pune, India \\ Dr. Shubhangee Ramaswami \\ Professor, Sinhgad Institute of Management \& Computer Application, \\ Pune, India

\section{Chaitanya Naidu} \\ Student, (MSc-CS), School of Computer Science, MIT-World Peace University, \\ Pune, India

\section{Nidhi Patel} \\ Student, (MSc-CS), School of Computer Science, MIT-World Peace University, \\ Pune, India
}

\begin{abstract}
Users nowadays have several web accounts for email, banking, blogging, social media, online shopping, and other services. The choice of passwords for these different web accounts poses a problem, resulting in a dilemma. Since users tend to have the same password for all of these accounts, if an attacker gains access to one of them, he will have access to all of the other user's accounts, including his banking passwords, email, and online shopping passwords. Users can have a propensity to use easy-toremember or weak passwords if they use different passwords for different systems, and there is also a high risk of users losing their passwords, raising the associated user support required for password resets. A secure password manager capable of storing multiple user accounts and passwords can be used to solve this issue, relieving users of the stress and challenge of remembering multiple accounts and passwords. To address this problem, an effective tool for managing password is designed and implemented.
\end{abstract}

Keywords: Encryption, Hashing, AES-256 Algorithm, password-protection, hash-key, Password Manager, Spoofing, Threats, master password, master-key. 
Cite this Article: Rajeshree Khande, Shubhangee Ramaswami, Chaitanya Naidu and Nidhi Patel, An Effective Mechanism for Securing and Managing Password using AES256 encryption \& PBKDF2, International Journal of Electrical Engineering and Technology (IJEET), 12(5), 2021, pp.1-7. https://iaeme.com/Home/issue/IJEET?Volume $=12 \&$ Issue $=5$

\section{INTRODUCTION}

In the digital era we are all dependent on information technology more than ever. All the business are happening though the internet and we all heavily dependent of information technology to access the e-commerce services thorough internet. Now a day many users have multiple account access the various internet services and it is quite difficult to remember the password for multiple account such as email, banking, blogging, forums, Social media, online shopping, cloud storage and other services. A challenge presents us with the choice of passwords for these various web accounts. The selection of passwords for these various web accounts presents us with a challenge. If each of these accounts uses a single password, if the attacker gains access to one of the accounts, the attacker gains access to the other user's accounts, including his bank passwords, email, online shopping passwords, and so on. But at the same time if bothered, users can have a preference to use easy-to-remember or weaker passwords as they choose different passwords for different systems which is also less secure.

Every individual need protection against various cyber-attacks and cyber security threats to protect their sensitive information. Cybercrime and malware are continuous extortions to anyone for digital presence. Each individual needs to protect their sensitive information to minimize high risk of information loss as it is very expensive. Information is the most significant to individual, it is the responsibility of each individual to protect it. When an individual anyone thinks of securing information, the first step is to create a strong password that is difficult to crack. Now a days many people becoming victim of cybercrimes such as phishing attacks, Password attack, Spear Phishing Attacks, Malware Attacks, Ransomware, Over the shoulder Attack, Sniffing Attack, Login Spoofing Attack, Identity theft and invasion of privacy. Following are the common security risk for information loss

- Over the shoulder Attack: As the name implies, a password can be obtained by indirect surveillance with a camera or by looking over one's shoulder. This is the simplest form of attack.

- Brute Force Attack: Since the passwords are limited to a finite number of alphanumeric characters, an attacker can use a powerful computer to test all possible combinations before identifying the correct password.

- Sniffing Attack: If a password is transmitted unencrypted over a network, it can be intercepted by network sniffing software, or an attacker can use a key logger to detect the password as it is typed.

- Login Spoofing Attack: The attacker creates a fake login screen that appears to be identical to the real one.

- If the user entered his or her credentials on that screen, the intruder would receive the password. 


\section{LITERATURE REVIEW}

\subsection{Verizon Data Breach Investigations Report (DBIR) 2019: Study says that $\mathbf{8 0 \%}$ of hacking-related breaches still tied to passwords}

Although the entire Verizon Data Breach Investigations Report (DBIR) 2019 report is worth reading, a few access and authentication-related findings in particular caught our attention this year.

Although the types of attacks and threats can vary from year to year, one constant remains: compromised passwords continue to be a major contributor to successful attacks. This detailed study examines security trends, investigates how data breaches occur, and offers security solutions for businesses. The 2019 DBIR evaluated 41,686 security incidents, with 2,013 (5\%) of them being reported data breaches, thanks to contributions from 73 data sources. They acknowledged that nothing has improved, with compromised and poor passwords still accounting for 80 percent of hacking-related breaches. Stolen credentials were used in 29 percent of all hacks, regardless of attack type. Even as more businesses invest in password protection, it's obvious that many are still having trouble handling passwords and avoiding password-related attacks. Password managers are explicitly listed in the DBIR as a key recommended tool in foiling hacking attempts, and the report concludes that password managers are a vital tool.

\subsection{Existing Password Managers: KeePass}

KeePass is a free open-source password manager that allows you to securely manage your passwords. All of your different account passwords are stored in a single folder, and you only need to remember one master password to access it all. KeePass is really free, and more than that it is open source and can export your passwords to a text file. But this password tool has few drawbacks as well. It takes time to understand for 'non-technical' types and does not provide web-based solution that support password auto-fill. Keepass only has a local database. There is no web application to log into, and password synchronization over the internet is not supported. A USB drive or other tools, such as Dropbox, may be used to share passwords. Also, it does not offer built built-in synchronization

\section{EFFECTIVE CLOUD-BASED MECHANISM FOR MANAGING PASSWORD}

To overcome this problem, in this paper, authors present the effective cloud-based mechanism to secure password of various account from malicious attacks by using Master password concepts. Passwords is the first layer of defense against unauthorized access to personal sensitive information. The stronger your password, the more protected your information and data from hackers and malicious software. Therefore, it recommended to have strong passwords for all accounts. Managing strong passwords is difficult for many people in the modern world. To solve this, this paper presents a cloud based effective tool for managing password. Because of its ease of use and implementation benefits, password security is an essential fundamental feature for information security that will not be replaced in the near future. Passwords are used as a first line of protection to protect almost all electronic data, including networks, servers, computers, accounts, databases, and so on. Despite its security flaws and vulnerabilities, textbased passwords are the most widely used authentication method. In order to create a functional and safe framework, design and implementation choices, as well as parameter evaluation, were crucial in the development of strong passwords. Authors designed and implemented effective cloud-based tool for managing password. This tool helps the user to keep multiple account password secure and user can access any of his or her account with master password. This solution offers multiple benefits over exiting password management tool such as 
- It is cloud-based solution, so user can synchronize their information across multiple computers and devices.

- Also, user can fill in not only passwords but also popular web forms automatically.

- This mechanism help user to store unique notes about the website or password with the application itself.

- It can be used regardless of the browser you are using.

- Browser extensions can help in auto-filling and ease of access.

- It uses combination of AES-CBC 256-bit and PBKDF2 encryption techniques to secure information. So, it is very difficult for attacker or hacker to gain access to sensitive information.

\section{METHODOLOGY}

Cybercriminals are constantly on the lookout for weak points that can be exploited to compromise security. How can users be sure that their data is secure, no matter where it is, in this increasingly linked digital era? To implement effective, difficulty to crack and more secure tool authors used the encryption algorithm AES-CBC 256-bit and Password-Based Key Derivation Function-2 (PBKDF2) hashing function.

\subsection{AES-CBC 256-bit Encryption}

Encryption is most common methods to safeguard sensitive information and data. The encryption mechanism takes plain text and converts it into cypher text, which is made up of random characters.

It can only be decrypted by those who have the special key. AES employs symmetric key encryption, which entails using only one secret key to encrypt and decrypt data. The Advanced Encryption Standard (AES) is the first and only publicly available cypher authorized for protecting top secret information by the US National Security Agency (NSA).

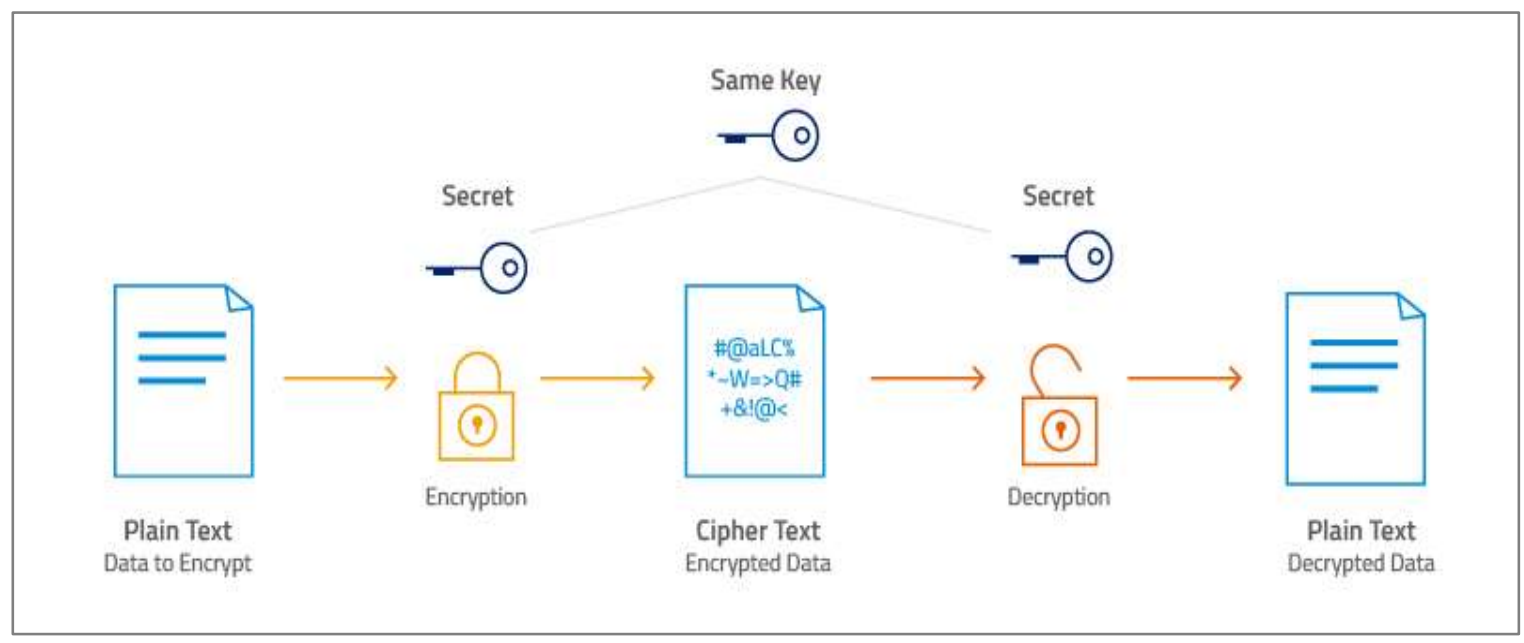

Figure 1 Symmetric key encryption

Source: www. google.com

AES-256, which has a key length of 256 bits, supports the largest bit size and is practically unbreakable by brute force based on current computing power, making it the strongest encryption standard. The AES-256 has $1.1 \times 10^{77}$ possible key combination. With proper implementation and a strong encryption key (master password), AES is considered 
unbreakable. User data is never sent to the cloud servers without first being encrypted on user's local device using AES 256-bit encryption.

\subsection{Hashing Algorithm: PBKDF2}

Password-Based Key Derivation Function 2 (PBKDF2) makes it harder for someone to determine Master Password by making repeated guesses in a brute force attack. PBKDF2 prevents password cracking software from making full use of graphics processing units (GPUs) which lower guess rates from hundreds of thousands to tens of thousands per second.

\section{PRACTICAL IMPLEMENTATION}

\subsection{Generation of Master Password}

Password managers are software programs that implement best practices for password generation and security (such as by using combination of encryption and hashing). The user may prompt the password manager to automatically pull the correct password from a database and authenticate into a system/software through form filling by using a master password/key. Password managers may be cloud-based, browser-based, or desktop-based.

When you first register, you are asked to make a master password. This master password is then appended to your email id and passed to a hashing function PBKDF2 to derive the encryption key from your master password. This key is then salted and hashed and is known as Authentication Key. The default iteration count used with PBKDF2 is 50,000 iterations on the client (this client-side iteration count is configurable from your account settings), and then an additional 50,000 iterations when stored on servers. After the key is salted and hashed, it is stored in the cloud and used for future authentication. Also, this newly generated key is again hashed to create a unique vault key which is used to encrypt and decrypt all the contents in the password manager. All the encryption and decryption happen in the client side so as to ensure that no unencrypted data is ever passed through the net or stored in the database. The purpose of the Authentication Key is to identify a particular user and retrieve the vault whereas the purpose of the Vault Key is to encrypt and decrypt data. Hashing the master password through this many iterations ensures that no one can ever guess our master password our reverse engineer it.

\subsection{Methodology for Random Password Generation in Password Manager}

The purpose of a random password generator is to produce random passwords that are difficult to guess and crack during attacks. It is critical that the password is random and not made up of patterns. When we use patterns in passwords, it's much easier to guess. Password cracker will effectively eliminate everything that doesn't match a predictable pattern. Generally, random passwords have several benefits over user-selected passwords, where security and anonymity are improved. This technique was designed to create a random password consisting of upperand lower-case letters, 0-9 digits and special characters. ( $\sim$ ! @\# $\left.\$ \wedge \& *_{-}++=\right)$, with fixed length. The Password Generator algorithm selects a random character from a random character list and forms a password that is a mixture of numbers, lower- \& upper-case letters and special characters. The entire character size is $75[13+10+26+26=62]$, which indicates 13 special characters, 10 digits ( 0 to 9), 26 upper-case letters and 26 lower-case letters. There are 75 possibilities of occurrence of each character in password. For example, our password length is 12 characters. So, the number of possible passwords will be: $75 \times 75 \times 75 \times 75 \times 75 \times 75 \times 75 \times 75 \times 75 \times 75 \times 75 \times 75=75^{\wedge} 12$ 


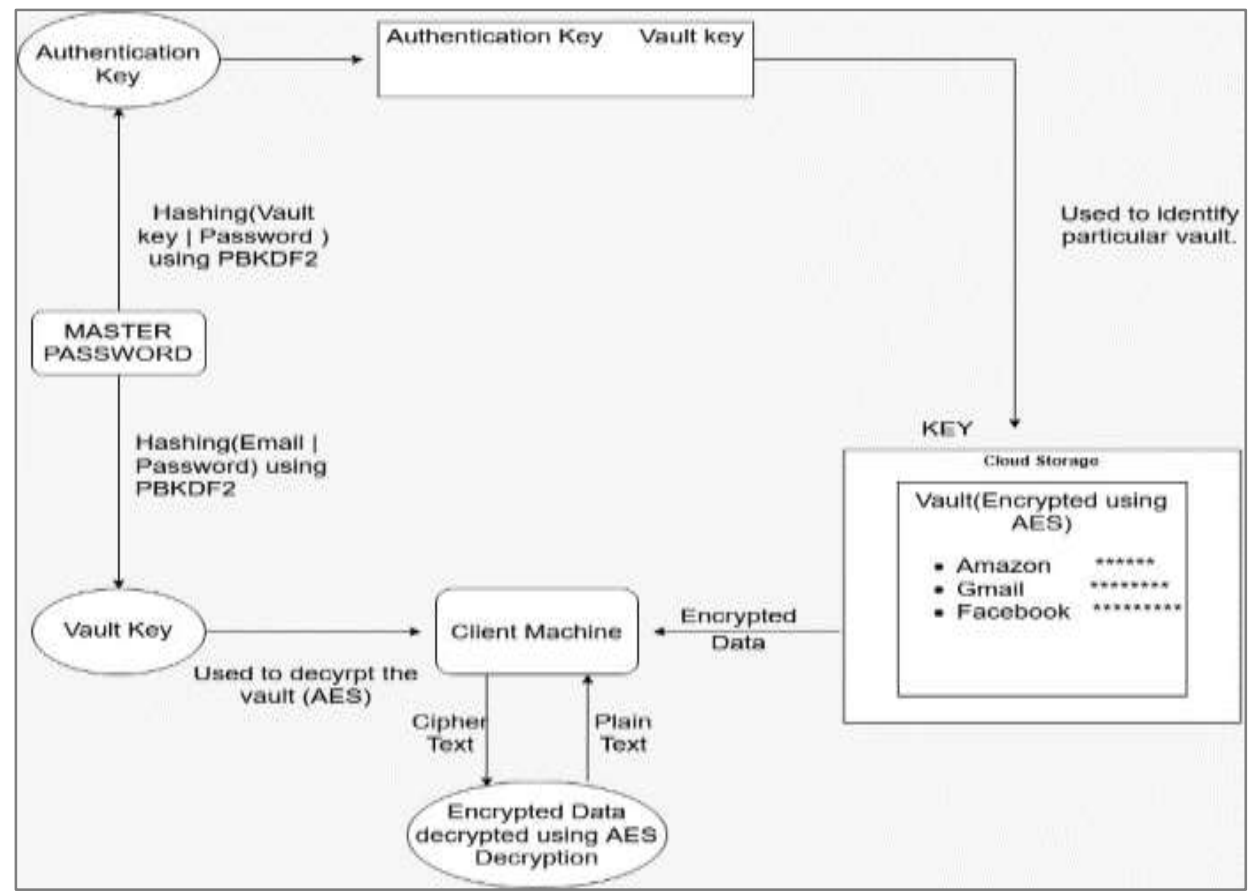

Figure 2 Working of cloud based Password manager

\section{CONCLUSION}

These days, password managers are the most underappreciated piece of software. People need them now more than ever, but they are unaware of their significance. Hacking attempts are increasing every day, and if nothing is done, they will continue to rise in the future. Organizations are doing everything possible to protect their servers from these attacks, but users are at risk because they use basic, easily cracked passwords. The users have been the target of several recent hacking attempts. Password managers are the only option for these users who can't recall complex passwords. Authors creating awareness about this importance of password security by developing effective solution to protect the user's sensitive information. Password managers are often proposed as a solution because they combine essential usability and security features. This tool remembers all of the users' passwords, so they don't have to remember them. This app assists users in entering passwords by automatically filling them into log-in forms, as well as assisting in the development of unique, random passwords. An attacker or hacker cannot steal password through network as it passes hash-key to client side and decrypt it at client side only. The data is encrypted using AES-256 encryption algorithm and PBKDF2 which is the current industry standard, additionally, the username and password are encrypted with a key generated from the user's master password, ensuring data security, and the password manager can be integrated into the browser as an extension, ensuring high compatibility and ease of use for end users.

\section{REFERENCES}

[1] Katha Chanda, "Password Security: An Analysis of Password Strengths and Vulnerabilities", I. J. Computer Network and Information Security, 2016, 7, 23-30

[2] Moshe Zviran and William J. Haga, "Password Security: An Empirical Study", Journal of Management Information Systems, Vol. 15, No. 4 (Spring, 1999), pp. 161-185 (25 pages) 
[3] M. Yıldırım \& I. Mackie "Encouraging users to improve password security and memorability", International Journal of Information Security volume 18, pages 741759(2019)

[4] Viktor Taneski, Marjan Heričko, Boštjan Brumen, "Systematic Overview of Password Security Problems", Acta Polytechnica Hungarica, Vol. 16, No. 3, 2019

[5] Moshe Zviran, William J. Haga," Password Security: An Empirical Study”, Journal of Management Information Systems, Volume 15, 1999 - Issue 4

[6] M.E. Thomson, R. von Solms, Information security awareness: educating your users effectively", Information Management \& Computer Security, Vol. 6 No. 4, pp. 167173. https://doi.org/10.1108/09685229810227649

[7] Rossouwvon SolmsJohanvan Niekerk, "From information security to cyber security", Computers \& Security”,Volume 38, October 2013, Pages 97-102

[8] https://www.atpinc.com/blog/what-is-aes-256-encryption

[9] https://enterprise.verizon.com/resources/reports/2017_dbir.pdf

[10] https://medium.com/@ kasunpdh/how-to-store-passwords-securely-with-pbkdf2 $204487 f 14 \mathrm{e} 84$

[11] https://en.wikipedia.org/wiki/Advanced_Encryption_Standard

[12] https://keepass.info/ 